\title{
EPISTEMOLOGIA EM CIÊNCIA DA INFORMAÇÃO: CENÁRIO INTERNACIONAL A PARTIR DA WEB OF SCIENCE
}

\author{
EPISTEMOLOGY IN INFORMATION SCIENCE: \\ INTERNATIONAL SCENARIO FROM WEB OF SCIENCE
}

\author{
Augiza Karla Boso Anselmoa \\ Rosângela Schwarz Rodrigues ${ }^{b}$ \\ Cezar Karpinskic
}

\begin{abstract}
RESUMO
Introdução: A publicação de artigos científicos é necessária para o avanço da ciência. Objetivo: O objetivo geral é analisar o cenário internacional da publicação científica sobre Epistemologia em periódicos da área de Ciência da Informação indexados pela Web of Science (WoS). Os objetivos específicos são a) identificar os títulos de periódicos com maior número de publicação sobre o tema, bem como sua tipificação editorial; b) relatar os tipos de acesso dos periódicos por país; c) apontar as palavraschave mais utilizadas e d) Identificar os epistemólogos mais utilizados no referencial teórico. Metodologia: O estudo foi caracterizado como exploratório, descritivo e quantitativo. Como procedimentos técnicos, fez-se uma pesquisa bibliográfica na principal coleção da WoS, utilizando a estratégia de busca: $\left(\mathrm{Tl}=\left(\right.\right.$ epistem $\left.^{*}\right)$ AND $S U=($ library and Information Science)). O período estipulado foi de 2003 a 2018 e obteve-se um total de 113 artigos válidos. Resultados: Os resultados mostram que os periódicos com mais artigos publicados foram Knowledge Organization, com 14 artigos, seguido do Journal of Documentation, com nove artigos. Observa-se que os 113 artigos foram publicados em 41 títulos de periódicos e que pertencem a 26 editoras distintas. As palavras-chave mais utilizadas foram "epistemology" citada 23 vezes, seguido de "information science", 10 vezes e "methodology", 04 vezes. Quanto aos epistemólogos mais citados, o destaque foi para Wittgenstein, seguido de Kuhn, Feyerabend e Popper. Conclusões: Conclui-se que o cenário internacional da publicação científica sobre Epistemologia em periódicos da área de Ciência da Informação, indexados pela Web of Science (WoS) relatam a evolução filosófica e teórica da própria epistemologia aonde, ainda, a grande maioria $(73,2 \%)$ são títulos por subscrição.
\end{abstract}

a Doutoranda do Programa de Pós-Graduação em Ciência da Informação da Universidade Federal de Santa Catarina (UFSC). Bibliotecária do Instituto Federal de Santa Catarina (IFSC). E-mail: augiza.ifsc@gmail.com

b Doutora em Engenharia de Produção pela Universidade Federal de Santa Catarina (UFSC). Professora do Programa de Pós-Graduação em Ciência da Informação da Universidade Federal de Santa Catarina (UFSC).E-mail: rosangela.rodrigues@ufsc.br

c Doutor em História pela Universidade Federal de Santa Catarina (UFSC). Professor do Programa de Pós-Graduação em Ciência da Informação da Universidade Federal de Santa Catarina (UFSC). E-mail: cezar.karpinski@gmail.com 
Descritores: Produção textual científica. Periódicos científicos. Epistemologia.

\section{INTRODUÇÃO}

Os avanços científicos ocorrem de maneira permanente na sociedade, de forma que o investigador faça parte da helicoide ${ }^{1}$ da pesquisa, uma vez que a publicação dos resultados em periódicos qualificados é a base para o progresso da ciência. Ademais, a ciência possui um sistema de comunicação próprio, em que os pesquisadores, as sociedades científicas, as universidades e os órgãos de regulação e avaliação de pesquisa utilizem periódicos a fim de darem visibilidade às novas investigações em um processo contínuo de retroalimentação do sistema (MERTON, 1973; ZIMAN, 1979, 1981).

Para tanto, os pesquisadores precisam buscar nos periódicos científicos qualificados de sua área, os trabalhos já publicados para que não haja o risco de duplicar estudos e ou usar metodologias impróprias, pois poderiam comprometer a própria realização da investigação (ABADAL, 2012). Com isso, a publicação e a divulgação dos resultados de seus estudos são as formas que o investigador encontra para registrar a originalidade e confirmar que o resultado obtido foi bom o suficiente para superar o ceticismo dos pares da comunidade (ZIMAN, 1979).

Como instituição social, a ciência é regida por um ethos, envolvendo valores e normas validadas e aceitas pelos pesquisadores (MERTON, 1973; MUELLER (1995). De acordo com Merton (1973), há quatro normas: Universalidade; Compartilhamento/comunalismo; Imparcialidade e desapego material; e Ceticismo. Na visão de Storer (1966), existem outras duas normas, propostas inicialmente por Barber: racionalidade e neutralidade emocional; e Ziman (1984) acrescenta a originalidade (WHITLEY, 1977).

Além de ser um canal de comunicação científica, o periódico tornou-se uma instituição social representativa da comunidade de pesquisadores de uma área onde os autores publicam em títulos selecionados que obtém prestígio,

\footnotetext{
${ }^{1}$ Guimarães (2009, p. 112) utiliza o termo "Helicoide informacional e não circular ou cíclico, uma vez que a informação produzida pelo sistema nunca será igual àquela que nele ingressou originalmente". O mesmo acontece com a helicoide da pesquisa, tendo em vista que após o feedback ou retroalimentação as informações/pesquisas sempre se modificam.
} 
reputação e reconhecimento dos pares e das instituições. (MERTON, 2013; LÓPEZ-COZAR, 2017; MARCOVICH; SHINN, 2013; WHITLEY, 1977).

López-Cozar (2017) destaca que o periódico se tornou o modo preferido dos cientistas com intuito de transmitir o conhecimento dos resultados de suas pesquisas e assumir o papel de registro e arquivo da ciência. Além disso, os periódicos científicos são instrumentos-chave na organização, na estruturação e na institucionalização social de todas as áreas do conhecimento, pois os artigos com resultados das pesquisas são o principal elemento constituinte do registro da produção e do uso do conhecimento científico.

Ao analisar a evolução da epistemologia na produção científica brasileira em Ciência da Informação, Francelin (2018, p. 92) explica que "a emergência de novos contextos paradigmáticos, conforme observado por Saracevic (1996), Pinheiro (1999), Aquino (2002), Capurro (2003) e Robredo (2003) pode ter sido uma das características que motivou o aumento das pesquisas (...)". Assim, se amplia o número de autores potenciais na área e a quantidade de publicações.

A estrutura e as formas pelas quais a comunicação é realizada desempenham um papel relevante no processo científico, desde a consulta aos registros das pesquisas já realizadas bem como a utilização em novas pesquisas até a comunicação e a divulgação dos resultados dessas para a comunidade. Francelin $(2018$, p. 91) reforça a ideia que

mapear a epistemologia na pesquisa pode ser o mesmo que tentar saber como o termo "epistemologia" é usado na Ciência da Informação. Se este é um caminho possível, então é aceitável dizer que o uso de um termo leva ao entendimento do seu significado. Portanto, quando se fala em pesquisar como o termo epistemologia está sendo usado, talvez seja o mesmo que perguntar pelo seu significado. $O$ uso de termos e de conceitos pode ser verificado através da análise do discurso e da linguagem ou mediante a aplicação de outros recursos metodológicos. Neste caso, os usos do termo "epistemologia" podem estar fundamentados, para além da palavra, em conhecimento conceitual, legitimado em atores, autores, disciplinas e pesquisa.

Dessa maneira, pesquisas epistemológicas podem ser facilmente difundidas e divulgadas aos cientistas e à sociedade, pois segundo Francelin (2018, p. 99) "a quantificação da produção científica fortalece a hipótese do interesse coletivo e espontâneo sobre o tema epistemologia na Ciência da 
Informação."

Ao discutir o conceito de "Epistemologia", Japiassu (1977, p. 11, grifo do autor) se diz "consciente de que falar de epistemologia, hoje, já é engajar-se num espaço polêmico ou conflitante, pois sob este título apresentam-se trabalhos que frequentemente nada têm de comum, quando não se excluem explicitamente". Embora essa constatação do autor seja da década de 1970, ela ainda é atual, haja vista que o contexto contemporâneo continua a demandar reflexões sobre os princípios, os valores e a razão de ser do que se produz em ciência e tecnologia. Além disso, pesquisar sobre epistemologia no campo da Ciência e não da Filosofia, como é a proposta deste artigo, é um desafio ainda maior, tendo em vista que o recorte que se exige para esta prática é sempre excludente.

Por isso, o debate acerca da epistemologia e da história das ciências é perene, contínuo e provocado a partir dos problemas e dos desafios do lugar de pesquisa do cientista. Nesse sentido, a Epistemologia é, essencialmente, "o estudo crítico dos princípios, das hipóteses e dos resultados das diversas ciências. Semelhante estudo tem por objetivo determinar a origem lógica (não psicológica) das ciências, seu valor e seu alcance objetivos" (JAPIASSU, 1977, p. 25, grifo do autor).

Na estrita relação entre "Epistemologia" e "Ciência", Chalmers (1993) destaca as principais correntes teóricas que, no Século XX, fundamentam o fazer científico. Nesse cenário, o autor ressalta a contribuição de epistemólogos como Paul Feyerabend, Karl Popper, Gaston Bachelard, Thomas Kuhn, Imre Lakatos, Willard van Orman Quine e Ludwig Wittgenstein. As reflexões destes autores formam um panorama epistemológico que, constituído no decorrer do Século $\mathrm{XX}$, continua fomentando discussões acerca dos fundamentos do conhecimento científico. Dessa forma, a categorização de Chalmers (1993) é atual e serve como ponto de partida para a identificação de correntes, autores e obras que despontam como referências no campo da Ciência da Informação. Em vista disso, apresenta-se a questão de pesquisa como sendo: Qual o cenário internacional da publicação científica sobre o tema "Epistemologia" em periódicos na área de Biblioteconomia e Ciência da Informação? Para responder a essa questão traz como objetivo geral analisar o cenário internacional da 
publicação científica acerca do tema "Epistemologia" em periódicos na área de Biblioteconomia e Ciência da Informação. Os objetivos específicos deste estudo são

a) identificar os títulos de periódicos com publicação sobre o tema epistemologia na área de Biblioteconomia e Ciência da Informação, entre os anos de 2003 a 2018, indexados na Web of Science (WoS) e sua tipificação editorial;

b) relatar os tipos de acesso dos periódicos da área de Biblioteconomia e Ciência da Informação sobre o tema epistemologia por país;

c) apontar as palavras-chave mais utilizadas nos artigos sobre o tema epistemologia na área de Biblioteconomia e Ciência da Informação, entre os anos de 2003 e 2018, indexados na WoS.

d) identificar os epistemólogos contemporâneos utilizados no referencial teórico.

\section{METODOLOGIA}

O estudo desenvolvido foi caracterizado como exploratório e descritivo com coleta de dados, em que não houve influências do autor no assunto pesquisado. (SAMPIERI; COLLADO; LUCIO, 2006; CRESWELL, 2010). Quanto à forma de abordagem do problema, pode-se caracterizar como sendo quantitativo com estatística descritiva e qualitativa.

No primeiro momento, para realizar esta pesquisa fez-se a coleta de dados na principal coleção da WoS, utilizando a estratégia de busca: $\left(T I=\left(e p i s t e m^{*}\right)\right.$ AND SU=(library and Information Science)); sendo que TI significa títulos dos documentos e SU é a área da pesquisa. Restringiu-se o resultado pelo tipo de documento, utilizando a opção: "article" (artigo), quanto aos idiomas optou-se por: "all languages" (todas as línguas). O período estipulado foi de 2003 a 2018. Dessa maneira, realizou-se a busca em julho de 2019 e recuperaram-se 113 artigos válidos. Conforme figura 1, sendo descartado um trabalho publicado em evento. 
Figura 1 - Estratégia de busca utilizada na Web of Science

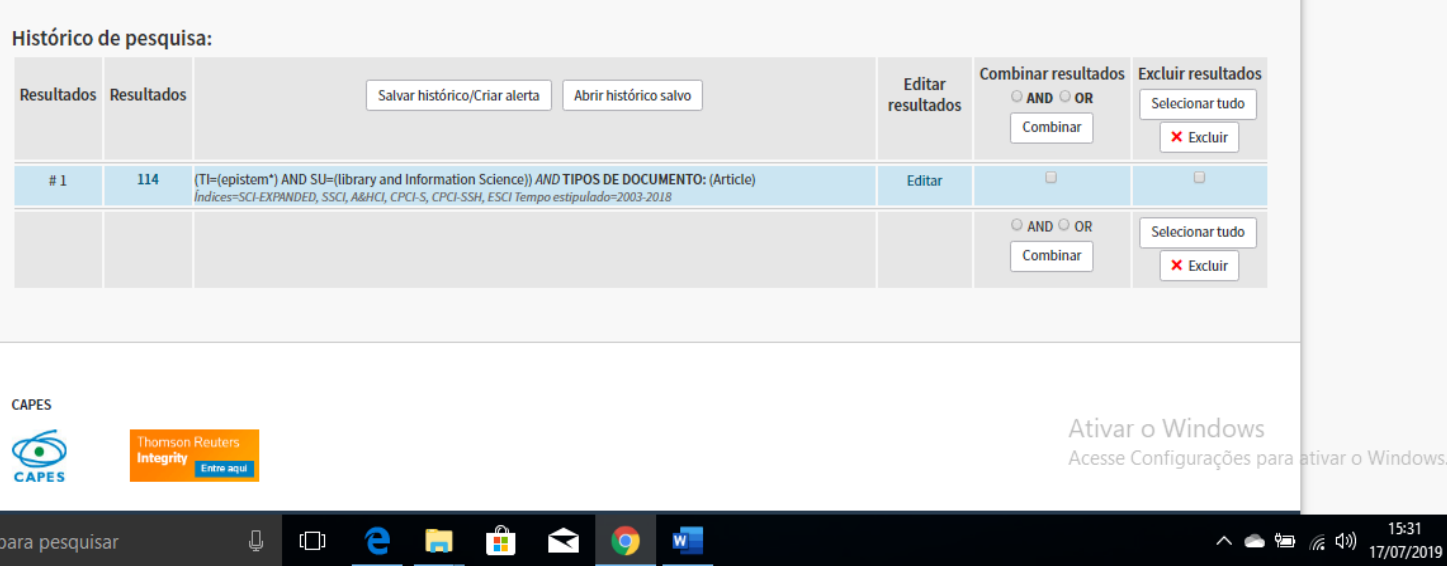

Fonte: Web of Science (julho de 2019)

A WoS foi escolhida por ser considerada a base em que estão os títulos de mais prestígio de todas as áreas do conhecimento, inclusive de Biblioteconomia e Ciência da Informação, assumindo assim que todos os periódicos e artigos atendam critérios de qualidade internacionalmente aceitos. O instrumento de coleta de dados foi uma ficha documental, elaborada no Microsoft Office Excel e os dados foram processados por meio de estatística descritiva.

No segundo momento da pesquisa, analisaram-se 4.954 referências utilizadas pelos autores dos 113 artigos. Nessa etapa, verificou-se quais foram os epistemólogos, as correntes filosóficas, o referencial teórico e obras mais utilizadas no referencial teórico. Para essa identificação, utilizou-se como base a classificação de Chalmers (1993) que destaca as principais correntes e perspectivas epistemológicas das ciências contemporâneas. Salienta-se que a análise referente aos dados das referências dos artigos da WoS é descritiva.

\section{RESULTADOS E DISCUSSÃO}

Os periódicos científicos surgiram, segundo Meadows (1999, p. 7), da crença de que para fazer novos descobrimentos era preciso que houvesse um debate coletivo. Entretanto, o motivo básico "encontrava-se na necessidade de comunicação, do modo mais eficiente possível, com uma clientela crescente interessada em novas realizações" (MEADOWS, 1999, p. 7). 
Define-se o Open Access ou Acesso aberto como a disponibilidade livre e gratuita dos conteúdos científicos e acadêmicos na internet. (ABADAL, 2012; SERNA-SAUCEDO; VILLANUEVA-VALADEZ, 2014; PINTO-SANTOS; VILLANUEVA-VALADEZ; CORTÉS-PEÑA, 2019; WAKELING et al., 2019).

Nesse contexto, para cumprir com o primeiro objetivo da pesquisa, identificaram-se os títulos de periódicos com maior número de publicações com a palavra Epistemologia no título na área de Biblioteconomia e Ciência da Informação, entre os anos de 2003 a 2018, indexados na Web of Science - WoS e sua tipificação editorial.

Dentre os 41 títulos de periódicos, obtiveram-se 15 títulos com três ou mais artigos publicados, o periódico que mais publicou foi Knowledge Organization com 14 artigos, seguido do Journal of Documentation com 9 e o Journal of the Associations for Information Science and Tecnology com 8, conforme apresentado no gráfico 1.

Gráfico 1 - Títulos de periódicos com três ou mais artigos publicados sobre 0 tema espistemologia, entre os anos de 2003 a 2018, indexados na WOS

Números de artigos

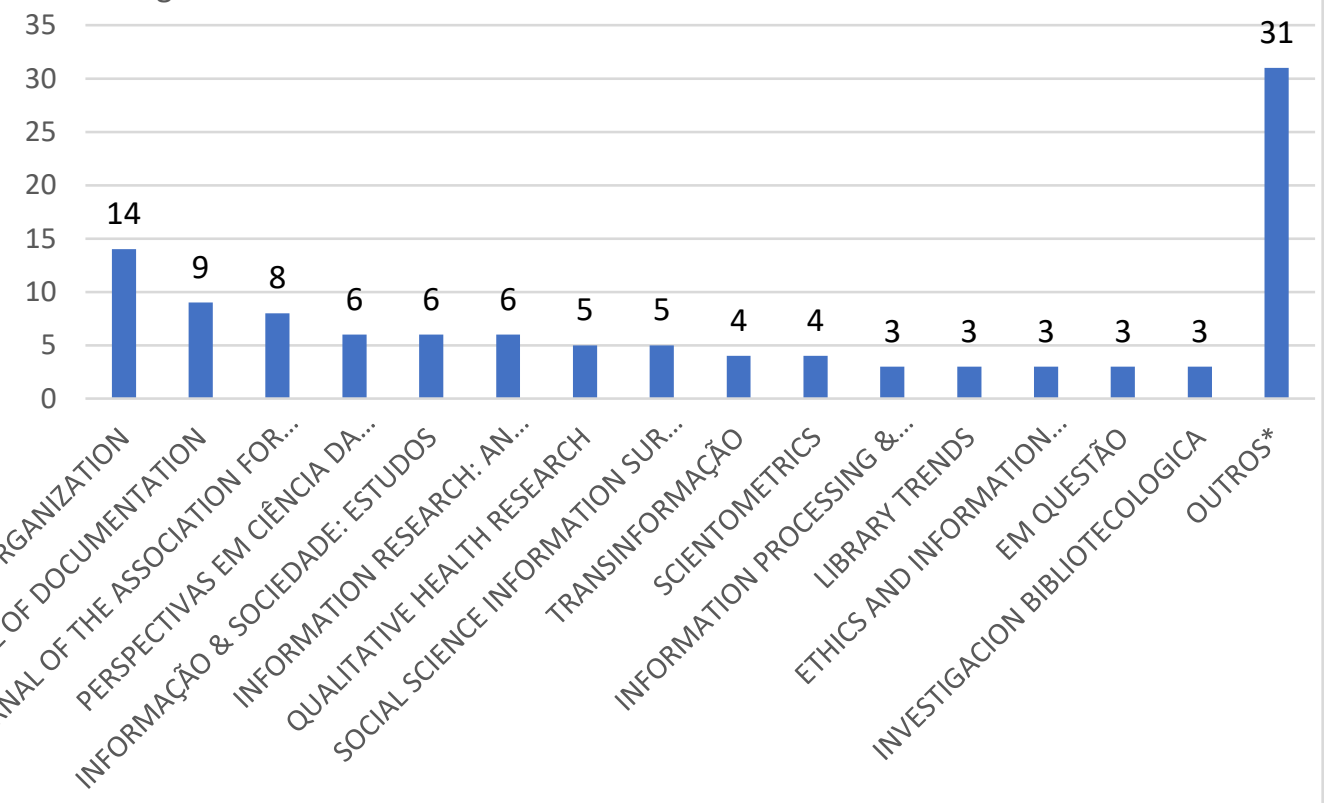

Fonte: Dados da pesquisa (2019)

Pode-se comparar com o estudo de Fico, Wasserman e Magalhães (2018, p. 287) na área de História os quais relatam que apenas $2 \%$ das revistas concentraram cerca de $30 \%$ de toda a produção. Esses resultados assemelham- 
se com os desta pesquisa na área de Biblioteconomia e Ciência da Informação, tendo em vista que os três títulos de periódicos ou 7,3\% das revistas com maior número de artigos representam $27,4 \%$ das publicações.

Esses dados vão ao encontro da segunda lei bibliométrica formulada em 1934 por Bradford, conhecida também como Lei da Dispersão ou lei de Bradford, a qual reflete sobre o conjunto de periódicos. Segundo Araújo (2006, p. 14),

[...] ordenando uma grande coleção de periódicos em ordem de produtividade decrescente relevante a um dado assunto, três zonas aparecem, cada uma contendo $1 / 3$ do total de artigos relevantes (a primeira zona contém um pequeno número de periódicos altamente produtivos, a segunda contém um número maior de periódicos menos produtivos, e a terceira inclui mais periódicos ainda, mas cada um com menos produtividade).

Nesse caso, aplicando a Lei de Bradford, verificou-se que a primeira zona, ou seja, os mais produtivos são compostos por três $(7,3 \%)$ periódicos (Knowledge Organization, Journal of Documentation e Journal of the Associations for Information Science and Tecnology) que publicaram 31 (27,4\%) dos artigos sobre tema. Esses são considerados os "periódicos-núcleo". Já a segunda zona, são compostos por $7(17,1 \%)$ periódicos que publicaram 36 (31,9\%) artigos e os 31 (75,06\%) periódicos restantes publicaram 46 (40,7\%) artigos. Os 31 títulos com menos artigos apresentam uma grande dispersão, uma vez que cinco periódicos publicaram três artigos cada um; cinco publicaram dois artigos cada e 21 periódicos, um artigo cada um.

Dentre os 113 artigos recuperados, observou-se ainda que foram publicados em 41 títulos de periódicos pertencentes a 26 editoras distintas. As 26 editoras foram tipificadas em quatro modalidades: Governamental, Associativa, Comercial e Universitária. Verificou-se que a modalidade universitária e comercial concentrou 11 editoras cada uma, seguida de editoras de associações com três e uma editora governamental.

Além disso, as 11 editoras universitárias publicaram 12 títulos de periódicos, e desses publicaram 37 artigos. Já as 11 editoras comerciais publicaram 25 títulos de periódicos e desses geraram 71 artigos. Salienta-se que somente uma editora comercial, Ergon-Verlag, responsável pelo título do periódico Knowledge Organization, responde por 14 artigos. A segunda editora 
comercial com maior número de artigos publicados foi a Sage Publications, com 13 artigos distribuídos em cinco periódicos e a terceira editora comercial foi a Emerald Group Publishing Limited com 11 artigos distribuídos em três títulos de periódicos. Com isso, os resultados apontam semelhanças com as pesquisas efetuadas por Coleman (2007) e por McGuigan e Russel (2008) no cenário norte americano que mostram a liderança de artigos publicados em periódicos de editoras comerciais, na maioria das áreas.

Toda essa dispersão de títulos segue o mesmo padrão em diferentes áreas, inclusive na Biblioteconomia e na Ciência da Informação, com as editoras comerciais com o maior número de periódicos, o que lhes permite ganho em escala e controle sobre o que é publicado.

As três editoras de associações, publicaram quatro artigos distribuídos em três periódicos e a única editora tipificada como governamental foi a Biblioteca Nacional de Cuba José Martí a qual possui um título de periódico, Bibliotecas: Anales de Investigación, com um artigo publicado.

Quanto ao tipo de acesso dos periódicos da área de Biblioteconomia e Ciência da Informação sobre o tema epistemologia por país, verificou-se que $73,2 \%$ dos periódicos possuem subscrição e os países que se destacaram foram EUA com $31,7 \%$ e Inglaterra com $24,4 \%$. Já no acesso aberto sem taxas, o Brasil lidera o ranking com 12,2\% das publicações (tabela 1).

Tabela 1 - Distribuição do tipo de acesso de periódicos da área de Biblioteconomia e Ciência da Informação sobre o tema epistemologia por país

\begin{tabular}{l|l|l|l|l|l|l|l|l}
\hline $\begin{array}{l}\text { Tipo de } \\
\text { Acesso }\end{array}$ & \multicolumn{2}{l}{$\begin{array}{l}\text { Acesso aberto } \\
\text { sem taxas }\end{array}$} & \multicolumn{2}{l|}{$\begin{array}{l}\text { Acesso aberto } \\
\text { com taxas }\end{array}$} & \multicolumn{2}{l|}{ Subscrição } & \multicolumn{2}{l}{ Total } \\
\hline País & $\mathrm{N}$ & $\%$ & $\mathrm{~N}$ & $\%$ & $\mathrm{~N}$ & $\%$ & $\mathrm{~N}$ & $\%$ \\
\hline EUA & 1 & 2,4 & 0 & 0 & 13 & 31,7 & 14 & 34,1 \\
\hline Inglaterra & 1 & 2,4 & 0 & 0 & 10 & 24,4 & 11 & 26,8 \\
\hline Brasil & 5 & 12,2 & 0 & 0 & 0 & 0 & 5 & 12,2 \\
\hline Holanda & 0 & 0 & 0 & 0 & 4 & 9,8 & 4 & 9,8 \\
\hline Canadá & 0 & 0 & 0 & 0 & 2 & 4,9 & 2 & 4,9 \\
\hline Cuba & 1 & 2,4 & 0 & 0 & 0 & 0 & 1 & 2,4 \\
\hline Alemanha & 0 & 0 & 0 & 0 & 1 & 2,4 & 1 & 2,4 \\
\hline México & 1 & 2,4 & 0 & 0 & 0 & 0 & 1 & 2,4 \\
\hline Espanha & 1 & 2,4 & 0 & 0 & 0 & 0 & 1 & 2,4 \\
\hline Suíça & 0 & 0 & 1 & 2,4 & 0 & 0 & 1 & 2,4 \\
\hline Total & 10 & 24,2 & 01 & 02,4 & 30 & 73,2 & 41 & 100
\end{tabular}

Fonte: Dados da pesquisa (2019) 
A identificação dos periódicos por país mostrou $43,8 \%$ do total de periódicos provenientes da Europa (Alemanha, 2,4\%; Espanha, 2,4\%; Holanda, 9,8\%; Inglaterra, 26,8\% e Suíça, 2,4\%), seguido da América do Norte com 41,4\% (Estados Unidos, 34,1\%; Canadá, 4,9\% e México, 2,4\%), América do Sul com 12,2\% (Brasil, 12,2\%) e América Central com 2,4\% (Cuba, 2,4\%). A predominância dos EUA com 14 títulos e da Inglaterra com 11 títulos, na publicação de artigos científicos é recorrente em todas as áreas do conhecimento (UNESCO, 2015). Verificam-se, no quadro 1, os títulos de periódicos, as editoras e o tipo de acesso por país.

\section{Quadro 1 - Editoras e a quantidade de artigos sobre epistemologia por títulos de periódicos, país da editora do periódico e tipo de acesso}

\begin{tabular}{|c|c|c|c|c|c|}
\hline Editora & Artigo & Título & País & Artigo & $\begin{array}{l}\text { Tipo de } \\
\text { acesso }\end{array}$ \\
\hline Ergon-Verlag & 14 & Knowledge Organization & $\mathrm{DE}$ & 14 & Subs * \\
\hline \multirow{5}{*}{ Sage Publications } & \multirow{5}{*}{13} & Qualitative Health Research & EU & 5 & Subs \\
\hline & & $\begin{array}{l}\text { Social Science Information Sur Les } \\
\text { Sciences Sociales }\end{array}$ & GB & 5 & Subs \\
\hline & & Information Development & GB & 1 & Subs \\
\hline & & Journal of Information Science & GB & 1 & Subs \\
\hline & & $\begin{array}{l}\text { IFLA Journal-international federation of } \\
\text { library associations }\end{array}$ & EU & 1 & Aberto \\
\hline \multirow{3}{*}{$\begin{array}{l}\text { Emerald Group } \\
\text { Publishing Limited }\end{array}$} & \multirow{3}{*}{11} & Journal of Documentation & GB & 9 & Subs \\
\hline & & Journal of Knowledge Management & GB & 1 & Subs \\
\hline & & $\begin{array}{l}\text { Program-Electronic Library and } \\
\text { Information Systems }\end{array}$ & GB & 1 & Subs \\
\hline \multirow{3}{*}{ Springer } & \multirow{3}{*}{9} & Scientometrics & $\mathrm{NL}$ & 4 & Subs \\
\hline & & Ethics and Information Technology & $\mathrm{NL}$ & 3 & Subs \\
\hline & & $\begin{array}{l}\text { International Journal of Computer- } \\
\text { supported collaborative learning }\end{array}$ & EU & 2 & Subs \\
\hline Wiley & 8 & $\begin{array}{l}\text { Journal of the Association for Information } \\
\text { Science and Technology }\end{array}$ & EU & 8 & Subs \\
\hline \multirow{5}{*}{ Elsevier } & \multirow{5}{*}{8} & Information Processing \& Management & GB & 3 & Subs \\
\hline & & Journal of Academic Librarianship & EU & 2 & Subs \\
\hline & & Library \& Information Science Research & EU & 1 & Subs \\
\hline & & Journal of Strategic Information Systems & $\mathrm{NL}$ & 1 & Subs \\
\hline & & Journal of Informetrics & $\mathrm{NL}$ & 1 & Subs \\
\hline \multirow{2}{*}{$\begin{array}{l}\text { Universidade Federal } \\
\text { de Minas Gerais } \\
\text { Universidade Federal } \\
\text { da Paraíba }\end{array}$} & 6 & Perspectivas em Ciência da Informação & BR & 6 & Aberto \\
\hline & 6 & Informação \& Sociedade: estudos & $\mathrm{BR}$ & 6 & Aberto \\
\hline University Sheffield & 6 & $\begin{array}{l}\text { Information Research - an international } \\
\text { electronic journal }\end{array}$ & GB & 6 & Aberto \\
\hline \multirow{2}{*}{$\begin{array}{l}\text { Johns Hopkins Univ } \\
\text { Press }\end{array}$} & \multirow{2}{*}{4} & Library Trends & GB & 3 & Subs \\
\hline & & Portal - Libraries and the Academy & EU & 1 & Subs \\
\hline $\begin{array}{l}\text { Pontifica } \\
\text { Universidade } \\
\text { Católica Campinas }\end{array}$ & 4 & Transinformação & BR & 4 & Aberto \\
\hline Taylor \& Francis & 3 & Cataloging \& Classification Quarterly & GB & 1 & Subs \\
\hline
\end{tabular}




\begin{tabular}{|c|c|c|c|c|c|}
\hline & & The Information Society & EU & 1 & Subs \\
\hline & & Journal of Health Communication & EU & 1 & Subs \\
\hline $\begin{array}{l}\text { Universidade Federal } \\
\text { do Rio Grande do Sul }\end{array}$ & 3 & Em Questão & $\mathrm{BR}$ & 3 & Aberto \\
\hline $\begin{array}{l}\text { Universidad Nacional } \\
\text { Autonoma Mexico }\end{array}$ & 3 & Investigacion Bibliotecologica & $M X$ & 3 & Aberto \\
\hline $\begin{array}{l}\text { Association } \\
\text { Computing } \\
\text { Machinery }\end{array}$ & 2 & $\begin{array}{l}\text { Data Base for Advances in Information } \\
\text { Systems }\end{array}$ & EU & 2 & Subs \\
\hline Blackwell Publishing & 2 & Information Systems Journal & $\mathrm{BG}$ & 2 & Subs \\
\hline $\begin{array}{l}\text { Oxford University } \\
\text { Press }\end{array}$ & 2 & Research Evaluation & GB & 2 & Subs \\
\hline $\begin{array}{l}\text { Associação dos } \\
\text { Servidores Arquivo } \\
\text { Nacional } \\
\end{array}$ & 1 & Acesso Livre & $\mathrm{BR}$ & 1 & Aberto \\
\hline $\begin{array}{l}\text { Biblioteca Nacional } \\
\text { Cuba Jose Marti }\end{array}$ & 1 & Bibliotecas - anales de investigacion & $\mathrm{CU}$ & 1 & Aberto \\
\hline \begin{tabular}{|l|} 
Canadian \\
Association \\
Information Science \\
\end{tabular} & 1 & $\begin{array}{l}\text { Canadian Journal of Information and } \\
\text { Library Science }\end{array}$ & CA & 1 & Subs \\
\hline IGI Global & 1 & $\begin{array}{l}\text { International Journal of Electronic } \\
\text { Government Research }\end{array}$ & EU & 1 & Subs \\
\hline Informs & 1 & Information Systems Research & EU & 1 & Subs \\
\hline $\begin{array}{l}\text { Multidiscipli- nary } \\
\text { Digital Publishing } \\
\text { Institute } \\
\end{array}$ & 1 & Publications & $\mathrm{CH}$ & 1 & Aberto \\
\hline $\begin{array}{l}\text { University Chicago } \\
\text { Press }\end{array}$ & 1 & Library Quarterly & EU & 1 & Subs \\
\hline $\begin{array}{l}\text { University Toronto } \\
\text { Press }\end{array}$ & 1 & Journal of Scholarly Publishing & CA & 1 & Subs \\
\hline University Zaragoza & 1 & $\begin{array}{l}\text { Scire-representacion y organizacion del } \\
\text { conocimiento }\end{array}$ & ES & 1 & Aberto \\
\hline
\end{tabular}

Fonte: Dados da pesquisa (2019).

Legenda: Subs $=$ Subscrição

Dos 113 artigos, 33 (29,20\%) deles estão em periódicos de acesso aberto e $80(70,80 \%)$ em periódicos com subscrições. O periódico Knowledge Organization permite o acesso aos artigos após três anos da publicação, mas conforme ASPESI et al. (2019), só é considerado Acesso Aberto a publicação imediata.

Verificou-se, ainda, que dentre os 80 artigos dos periódicos com subscrições nove estavam com livre acesso, sendo estes:

a) 1 artigo do Journal of Documentation dentre os 09 analisados nesta pesquisa;

b) 1 da Scientometrics dentre os 04 analisados;

c) 1 da Library Trends dentre os 03 analisados;

d) 1 da Research Evaluation dentre os 02 analisados na pesquisa; 
e) 2 da Date Base for Advances in Information Systems dentre os 2 analisados;

f) 1 do Portal - Libraries and the Academy dentre o único analisado.

No entanto, estes não foram considerados acesso aberto, pois segundo Aspesi et al (2019) o periódico não poderia ser fechado, o que leva a discussões sobre periódicos híbridos, não sendo o foco desse artigo.

Appel e Albagli (2019, p. 193) ressaltam que

o acesso aberto tornou-se, assim, um negócio lucrativo para as editoras comerciais, aumentando não apenas suas rendas, mas também o controle sobre todo o ciclo da comunicação científica [...]. Ressalta-se que este não era o modelo originalmente concebido nas diretrizes do movimento pelo acesso aberto, em que se vislumbrava a transição de periódicos por assinatura para o acesso aberto, seja pela via verde (dos repositórios), seja pela via dourada sem cobranças de taxas e assinaturas, pelo menos não nos elevados preços que viriam a ser praticados.

Verificou-se, ainda, que as editoras comerciais são as que mais publicam artigos sobre o tema, como visto no Quadro 1, em que Ergon-Verlag publicou 14 artigos, Sage Publications, 13; Emerald Group Publishing Limited, 11; Springer, 09; Wiley, 08 e Elsevier também com oito publicações.

Para estudar o vínculo institucional dos autores, optou-se por verificar a afiliação do primeiro autor do artigo. Observaram-se as instituições que obtiveram duas ou mais publicações de artigos por seus afiliados. Tiveram um total de 11 instituições que publicaram dois ou mais artigos, sendo publicado no total 27. (Gráfico 2). Salienta-se que houve 80 instituições que publicaram somente uma vez e seis autores sem vínculo institucional identificado. 


\section{Gráfico 2 - Vínculo institucional do $1^{\circ}$ autor do artigo sobre epistemologia, indexado na WoS, com duas ou mais publicações}

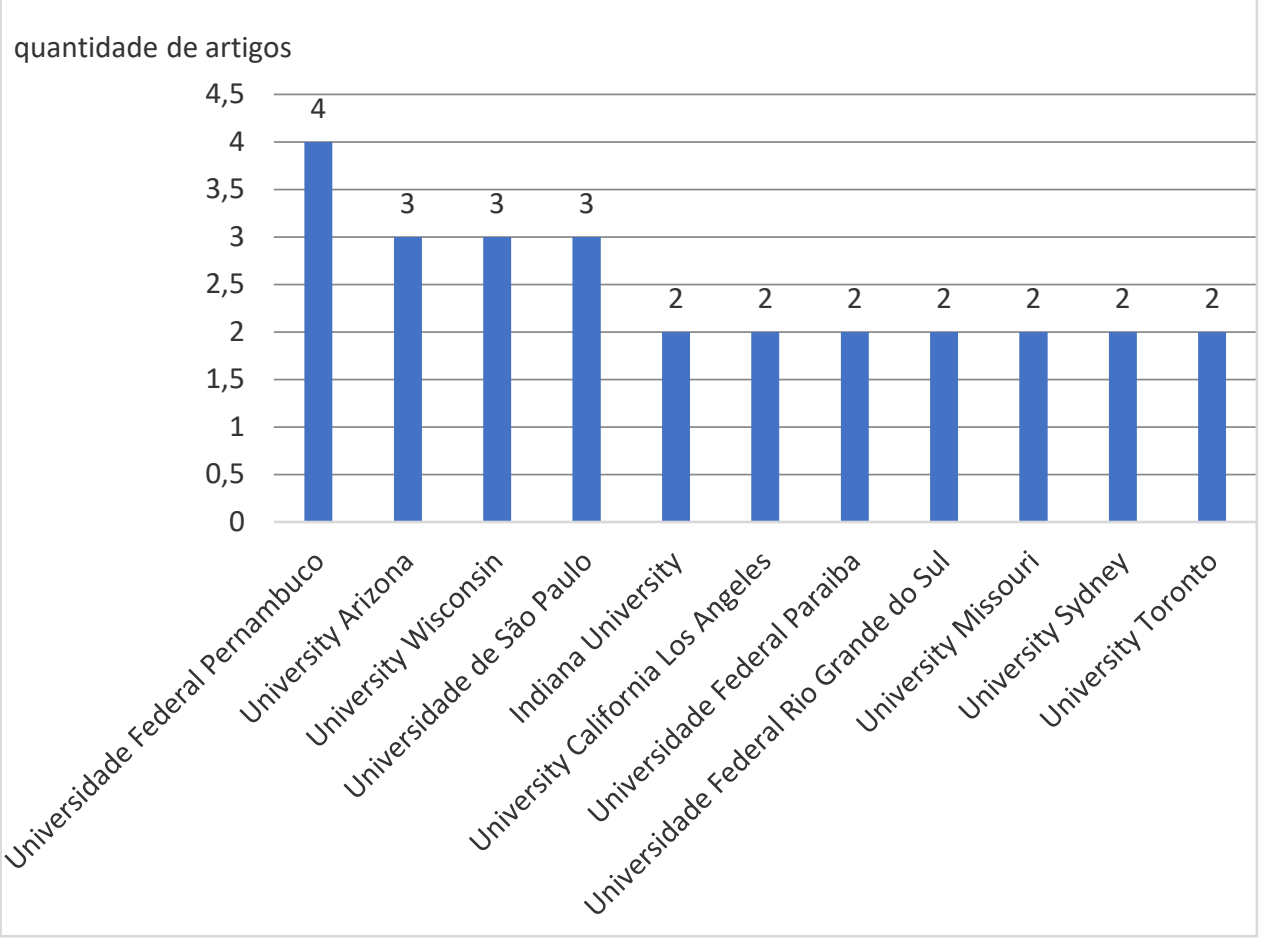

Fonte: Dados da pesquisa (2019)

Observou-se que as instituições brasileiras possuem destaques em quantidade de publicações, tendo em vista que das 11 instituições com dois ou mais artigos, quatro são brasileiras: Universidade Federal de Pernambuco (UFPE), quatro artigos publicados; Universidade de São Paulo - USP, três; Universidade Federal da Paraíba (UFPB) e a Universidade Federal do Rio Grande do Sul (UFRGS), ambas com dois artigos indexados na WoS. Verificouse, no quadro 2, os autores das 11 instituições que mais publicaram. As referências destas publicações encontram-se no apêndice A.

Observa-se que Silveira, M. A. A, vinculado à UFPE teve quatro publicações, todos em colaboração com Caregnato, S. A.; seguido de Budd, J. M. e Whitmire, ambos com duas publicações cada um e afiliados, respectivamente, à Universidade de Missouri e à Universidade de Wisconsin. Ademais, Budd realizou uma publicação com autoria única e uma em colaboração com Dumas, C. e Whitmire fez suas duas publicações em autoria única. 


\section{Quadro 2 - Primeira afiliação do primeiro autor por quantidade de artigos publicados}

\begin{tabular}{|l|l|c|l|}
\hline 1 a Autoria & Afiliação do 10 autor & $\begin{array}{c}\text { Artigo } \\
\text { N }\end{array}$ & Colaboradores \\
\hline Silveira, M.A.A. & Pernambuco & 4 & Caregnato, SE \\
\hline Budd, J.M. & University Missouri & 2 & $\begin{array}{l}01 \text { autoria única e 01 em } \\
\text { colaboração com: Dumas, C. }\end{array}$ \\
\hline Whitmire, E. & University Wisconsin & 2 & - \\
\hline $\begin{array}{l}\text { Bezerra, } \\
\text { M.A.A. }\end{array}$ & Universidade Federal da & 1 & Araújo, E.A. \\
\hline Brasileiro, F.S. & Universidade Federal da & 1 & $\begin{array}{l}\text { Loureiro, J.M.M. e } \\
\text { Preire, G.H.D. }\end{array}$ \\
\hline Carter, S.M. & University Sydney & 1 & Little, M. \\
\hline Choo, C.W. & University Toronto & 1 & - \\
\hline Coradini, O.L. & Universidade Federal do Rio & 1 & - \\
\hline Demarest, B. & Indiana University & 1 & Sugimoto, C.R. \\
\hline Fallis, D. & University Arizona & 1 & - \\
\hline Francelin, M.M. & Universidade de São Paulo & 1 & - \\
\hline Fricke, M. & University Arizona & 1 & - \\
\hline Furlan, P.K. & Universidade de São Paulo & 1 & Laurindo, F.J.B. \\
\hline Furner, J. & University California & 1 & - \\
\hline Goertz, G. & University Arizona & 1 & Mahoney, J. \\
\hline Hartel, J. & University Toronto & 1 & - \\
\hline Lee, H.L. & University Wisconsin & 1 & - \\
\hline Michell, J. & University Sydney & 1 & - \\
\hline Mostafa, S.P. & Universidade de São Paulo & 1 & - \\
\hline Semeler, A.R. & Universidade Federal do Rio & 1 & Pinto, A.L.; Vianna, W.B. \\
\hline Svenonius, E. & University California & 1 & - \\
\hline Zhang, G. & Indiana University & 1 & Jacob, E.K. \\
\hline
\end{tabular}

Fonte: Dados da pesquisa (2019)

A Universidade de São Paulo (USP) contou com três publicações:

Francelin, M. M. (autoria única); Mostafa, S. P (autoria única) e Furlan, P. K., este em colaboração com Laurindo, F. J. B.

A Universidade da Paraíba contou com duas publicações: Bezerra, M. A. A. em colaboração com Araújo, E. A.; e Brasileiro, F. S. em colaboração com e Loureiro, J. M. M. e Freire, G. H. D.

Já a Universidade Federal de Rio Grande do Sul contou com duas publicações: Coradini, O. L. (autoria única) e Semeler, A. R. em colaboração com Pinto, A. L. e Vianna, W. B. 
Para apontar as palavras-chave mais utilizadas nos artigos sobre o tema epistemologia na área de Biblioteconomia e de Ciência da Informação, entre os anos de 2003 e 2018, indexados na WoS optou-se por fazer análise das palavras-chave do autor, denominado na WoS por DE, em que o próprio autor insere as palavras-chave que acha mais adequada para o artigo.

Verificou-se que dentre os 113 artigos indexados na WoS sobre epistemologia na área de Biblioteconomia e Ciência da Informação, entre os anos de 2003 e 2018, apenas 76 possuíam palavras-chave, que apresentaram um total de 399 termos.

Percebe-se que a palavra-chave mais utilizada foi "epistemology" citada 23 vezes, em segundo lugar, citado 10 vezes foi o termo "information Science", seguido de "methodology" com quatro vezes. Obtivemos um total de nove palavras-chave citadas três vezes cada uma, sendo elas: Classification, discourse analysis, information technology, interdisciplinarity, interpretative research, knowledge organization, ontology, social science e citation studies. Houve 29 palavras-chave que foram citadas duas vezes e 277 palavras-chave que foram mencionadas apena uma vez. Observou-se o aumento da produção científica epistemológica, principalmente nos últimos quatro anos (2015 a 2018).

A análise das palavras-chave mostra que a produção científica sobre Epistemologia em Ciência da Informação é tematicamente diversa. Vários são os assuntos que possibilitam a aproximação com o viés epistemológico da área, o que demonstra um sistema plural, interdisciplinar e aplicado às temáticas recorrentes da área. Como exemplo, destacam-se estudos voltados à Organização do Conhecimento (por meio de termos como classificação e ontologias), à Tecnologia e à Produção Científica.

Do ponto de vista quantitativo, a análise dos dois termos mais citados, "epistemology" e "information science" pode indicar que a área procura uma epistemologia específica para seu campo. Desse modo, tratar de Epistemologia em Ciência da Informação pode se referir a aproximações entre as abordagens clássicas da Epistemologia e as temáticas ou técnicas próprias do campo científico. Esse dado se reforça na relação com a terceira palavras-chave mais citadas que foi "methodology". 
Apesar de citada por apenas quatro artigos, é importante notar que, no aspecto quantitativo, a comunidade científica aproxima as reflexões epistemológicas ao contexto metodológico da Ciência da Informação. Entendese que este possa ser um indício de que ao tratarem de assuntos teóricos e/ou filosóficos comumente atrelados aos aspectos epistemológicos, os autores assumem uma perspectiva prática e aplicada. Dessa forma, reforçam-se as constatações de Japiassu (1977) de que a Epistemologia serve para a ciência como um instrumental das reflexões acerca dos princípios e dos sentidos do "fazer" científico, auxiliando no processo de aproximação entre teoria e prática, entre filosofia e ciência.

Para responder ao quarto objetivo específico, fez-se uma análise das referências de epistemólogos utilizados nos artigos, essa serviu como base para o panorama de correntes, autores e obras apresentado por Chalmers (1993). As referências utilizadas nos artigos são plurais e não se limitam a autores clássicos da área de Epistemologia. Isso porque das 4.954 referências dos artigos, apenas 75 se referem a epistemólogos, conforme se verifica na tabela 2.

Tabela 2 - Relação de epistemólogos citados pelos autores

\begin{tabular}{|c|c|}
\hline Autor & Referências \\
\hline Wittgenstein, Ludwig & 23 \\
\hline Kuhn, Thomas & 19 \\
\hline Feyerabend, Paul & 11 \\
\hline Popper, Karl & 10 \\
\hline Lakatos, Imre & 07 \\
\hline Quine, Willard van Orman & 04 \\
\hline Bachelard, Gaston & 01 \\
\hline Total & 75 \\
\hline
\end{tabular}

Fonte: Elaborado pelos autores a partir de Chalmers (1993) e dos dados da Pesquisa

$\mathrm{Na}$ análise das obras referenciais de epistemologia, destaca-se a influência da Lógica e da Filosofia da Linguagem, temáticas presentes na produção de Ludwig Wittgenstein, citado 23 vezes e Willard van Orman Quine, citado quatro vezes. Este defende uma "epistemologia naturalizada" que propõe transferir para a ciência as questões tratadas pela filosofia. (DUTRA, 2010). Já 
aquele se apresenta com posições filosóficas complexas relacionadas à lógica da linguagem, primeiramente, e à constituição de uma Filosofia da Linguagem, num segundo momento de sua vida e obra. Conforme as considerações iniciais do "Tractatus Logico-Philosophicus", pode-se dizer que este filósofo se perguntou sobre 'a lógica da linguagem' como problema do conhecimento e, por extensão, da teoria do conhecimento (WITTGENSTEIN, 1994).

De acordo com Thomas Kuhn, o segundo mais citado, instaurou-se o que Chalmers (1993, p.122) denominou como "relativismo na ciência" ao defender a influência do contexto histórico e das "características sociológicas das comunidades científicas". De certa forma, essa teoria apresenta convergências e divergências com outros dois epistemólogos também citados nos artigos analisados, Imre Lakatos e Karl Popper. Ainda de acordo com Chalmers (1993), Lakatos defende que as teorias epistemológicas devam ser analisadas como estruturas organizadas a partir de programas de pesquisa científica.

Popper é o autor que, em última instância, possibilita a construção epistemológica de Kunh e Lakatos. Por mais que suas teorias divirjam em vários aspectos, sendo resguardadas as devidas distinções no que convergem, pois foi a posição de Popper contra o empirismo lógico que possibilitou um pensamento epistemológico crítico a partir da segunda metade do século XX. Nas palavras de Japiassu (1977, p.106) "[...] a epistemologia de Popper pode caracterizar-se como uma crítica constante às concepções científicas já existentes, tentando sempre instaurar novas hipóteses ou conjecturas ousadas [...]".

Nesse sentido, pode-se também entender como 'ousada' a proposta epistemológica de Paul Feyerabend citado 10 vezes pelos autores de epistemologia em Ciência da Informação. A defesa de uma "teoria anarquista do conhecimento" faz de Feyrabend um dos autores que melhor reflete a dificuldade de se encontrar consensos na epistemologia contemporânea. Isso porque, de acordo com Chalmers (1993), Feyrabend

[...] argumenta de forma convincente que as metodologias da ciência fracassaram em fornecer regras adequadas para orientar as atividades dos cientistas. Além dos mais ele sugere que, dada a complexidade da história, é extremamente implausível esperar que a ciência seja explicável com base em algumas poucas regras metodológicas simples. (CHALMERS, 1993, p.173) 
Por fim, o quadro de epistemólogos citados ainda contempla Gaston Bachelard que, de acordo com Japiassu (1977, p. 73), possibilita a emergência de uma "epistemologia histórica", uma vez que "a epistemologia é indissociável da história das ciências, quer no aspecto 'sancionado' (história daquilo que é científico na prática científica), quer em seu aspecto 'superado' (história do 'não científico' na prática das ciências)".

\section{CONSIDERAÇÕES FINAIS}

Neste artigo, analisou-se o cenário internacional da publicação científica sobre o tema epistemologia nos artigos de periódicos na área de Biblioteconomia e ciência da informação indexados na WoS, no período compreendido entre 2003 e 2018. Os 113 artigos recuperados foram publicados em 41 títulos de periódicos pertencentes a 26 editoras distintas.

Os títulos de periódicos com mais publicação sobre o tema epistemologia na área de Biblioteconomia e Ciência da Informação, entre os anos de 2003 a 2018, indexados na Web of Science - WoS foram Knowledge Organization com 14 artigos, seguido do Journal of Documentation com nove e o Journal of the Associations for Information Science and Tecnology com oito. Já as 26 editoras foram tipificadas em quatro modalidades: Governamental, Associativa, Comercial e Universitária, com isso se verificou que a modalidade universitária e comercial concentraram 11 editoras cada uma, seguida de editoras de associações com três e uma na editora governamental.

Quanto ao tipo de acesso dos periódicos da área de Biblioteconomia e Ciência da Informação acerca do tema epistemologia por país, observou-se que $73,2 \%$ dos periódicos possuem subscrição e os países que se destacaram foram EUA, com $31,7 \%$, e Inglaterra com $24,4 \%$. Já no acesso aberto, o Brasil lidera o ranking com $12,2 \%$ das publicações.

A palavra-chave mais utilizada foi "epistemology" citada 23 vezes; em segundo lugar, citado 10 vezes foi o termo "information Science", seguido de "methodology" com quatro vezes. É importante salientar que, nesse aspecto, a produção científica sobre Epistemologia em Ciência da Informação é tematicamente diversa e aplicada. 
Quanto às análises em relação às referências dos artigos, essas demonstraram que, no âmbito da Epistemologia contemporânea, a produção científica é plural e não se limita aos autores clássicos, uma vez que mostra uma área eclética que constitui referências específicas sobre o tema. No entanto, todos os epistemólogos contemporâneos destacados por Chalmer (1993) foram citados, sendo que Ludwig Wittgenstein, Thomas Kuhn e Paul Feyerabend receberam mais atenção da produção científica pesquisada.

Considerando a contribuição epistemológica dos autores mais citados, infere-se que, na produção analisada no que se refere especificamente à temática de Epistemologia, a área de Ciência da Informação enfatiza discussões sobre a "Linguagem" na temática de Wittgenstein; "Paradigmas e Estruturas das Ciências" na temática de Kuhn e "Teoria crítica das metodologias" na temática de Feyerabend. Entretanto, não se pode deixar de notar a influência de autores de prestígio no cenário epistemológico contemporâneo, como é o caso de Popper, Lakatos, Quine e Bachelard.

Assim, chegou-se à conclusão de que o cenário internacional de publicação científica sobre Epistemologia em periódicos da área de Ciência da Informação, indexados pela Web of Science (WoS) relatam a evolução filosófica e teórica da própria epistemologia e que, ainda, há a predominância de títulos (30) por subscrição.

\section{REFERÊNCIAS}

ABADAL, E. Acceso abierto a la ciencia. Barcelona: Editorial UOC, 2012.

APPEL, A. L.; ALBAGLI, S. Acesso aberto em questão: novas agendas e desafios. Inf. \& Soc.: Est., João Pessoa, v. 29, n. 4, p. 187-208, out./dez. 2019. Disponível em: https://periodicos.ufpb.br/ojs2/index.php/ies/article/view/50113/29300. Acesso em: 08 jan. 2020.

ARAÚJO, C. A. Bibliometria: evolução histórica e questões atuais. Em Questão, Porto Alegre, v. 12, n. 1, p. 11-32, jan./jun. 2006. Disponível em: http://www.brapci.inf.br/index.php/article/download/10036. Acesso em: 26 nov. 2019. 
ASPESI, C.; ALLEN, N.; CROW, R.; DAUGHERTY, S.; JOSEPH, H.; MCARTHUR, J.; SHOCKEY, N. SPARC landscape analysis: the changing academic publishing industry: implications for academic institutions. Washington: SPARC, 2019. Disponível em:

https://digitalcommons.unl.edu/scholcom/99/. Acesso em: 05 jun. 2020.

CHALMERS, A. F. O que é ciência afinal? São Paulo: Brasiliense, 1993.

COLEMAN, A. Self-archiving and the Copyright Transfer Agreements of ISIranked library and information science journals. Journal of the American Society for Information Science and Technology. v. 58, n. 2, p. 286-296, 2007. Disponível em: https://doi.org/10.1002/asi.20494. Acesso em: 05 ago. 2018.

CRESWELL, J. W. Projeto de pesquisa: métodos qualitativo, quantitativo e misto. 3. ed. Porto Alegre: ARTMED, 2010.

DUTRA, L. H. de A. Introdução à Epistemologia. São Paulo: Editora. UNESP, 2010. $190 \mathrm{p}$.

FICO, C.; WASSERMAN, C.; MAGALHÃES, M. S. Expansão e avaliação da área de história: 2010-2016. História da Historiografia, v. 11, n. 28, set/dez., 2018, p. 267-302. Disponível em: https://www.researchgate.net/publication/329591033 HISTORIA DA HISTORI OGRAFIA 267. Acesso em: 05 ago. 2019.

FRANCELIN, M. M. Epistemologia da ciência da informação: evolução da pesquisa e suas bases referenciais. Perspectivas em Ciência da Informação, v. 23, n. 3, p. 89-103, 2018. Disponível em:

http://hdl.handle.net/20.500.11959/brapci/93365. Acesso em: 01 ago. 2019.

GUIMARÃES, J. A. C. Abordagens teóricas de tratamento temático da informação: catalogação de assunto, indexação e análise documental. Ibersid, 2009. p. 105-117. Disponível em:

https://www.ibersid.eu/ojs/index.php/ibersid/article/view/3730. Acesso em: 23 maio de 2019.

JAPIASSU, H. F. Introdução ao Pensamento Epistemológico. Rio de Janeiro: Francisco Alves, 2. ed., 1977. 202 p.

LÓPEZ-COZAR, E. D. Evaluar revistas científicas: un afán com mucho presente y passado e incerto futuro. In: ABADAL, E. (Org.). Revistas científicas: situación actual y retos de futuro. Barcelona: Editora da Universidade de Barcelona, 2017.

MARCOVICH, A.; SHINN, T. Robert K. Merton, fundador da sociologia da ciência: comentários, insights, críticas. In: MERTON, R. K. Ensaios de sociologia da ciência. São Paulo: Associação Filosófica Scientiae Studia; Ed. 34, 2013. p. 253-273. 
MCGUIGAN, G. S.; RUSSEL, R. D. The business of academic publishing: a strategic analysis of the academic journal publishing industry and its impact on the future of scholarly publishing. Electronic Journal of Academic and Special Librarianship, v. 9, n. 3, 2008. Disponível em: http://southernlibrarianship.icaap.org/content/v09n03/mcguigan g01.html. Acesso em: 05 ago. 2019.

MEADOWS, A. J. A comunicação científica. Brasília: Briquet de Lemos, 1999.

MERTON, R. K. Ensaios de sociologia da ciência. São Paulo: Associação Filosófica Scientiae Studia; Ed. 34, 2013.

MERTON, R. K. The Sociology of Science: theoretical and empirical investigations. Chicago: University of Chicago Press, 1973.

MUELLER, S. P. M. O crescimento da ciência, o comportamento científico e a comunicação científica: algumas reflexões. Revista da Escola de

Biblioteconomia da UFMG, v. 24, n. 1, p. 63-84, 1995. Disponível em: https://brapci.inf.br/index.php/article/view/0000002743. Acesso em: 18 abr. 2019.

PINTO-SANTOS, A. R.; VILLANUEVA-VALADEZ, C.; CORTÉS-PEÑA, O. Percepciones del docente universitario sobre la comunicación científica de libre acceso. Revista Española de Documentación Científica, v. 42, n. 2, p. 01 15, 2019. Disponível em: https://doi.org/10.3989/redc.2019.2.1588. Acesso em: 12 jun. 2019.

SAMPIERI, R. H.; COLLADO, C. F.; LUCIO, P. B. Metodologia de Pesquisa. São Paulo: McGraw-Hill, 2006.

SERNA-SAUCEDO, N.; VILLANUEVA-VALADEZ, C. Implementación del acceso abierto al conocimiento y repositorio institucional UANL. In: TICAL, 2014. Anais [...], Disponível em: http://eprints.uanl.mx/4062. Acesso em: 12 jun. 2019.

STORER, N. The social system of Science. New York: Rinehardt e Winston, 1966 .

UNESCO. Unesco Science Report. Paris: United Nations Educational, Scientific and Cultural Organization. 2015. Disponível em: https://en.unesco.org/usr-contents. Acesso em: 15 ago. 2019.

WAKELING, S.; CREASER, C.; PINFIELD, S.; FRY, J.; SPEZI, V.; WILLETT, P.; PARAMITA, M. Motivations, Understandings, and Experiences of OpenAccess Mega-Journal Authors: Results of a Large-Scale Survey. Journal of the Association for Information Science and Technology, v. 7, n. 7, p. 754-768, 2019. Disponível em: https://onlinelibrary.wiley.com/doi/epdf/10.1002/asi.24154. Acesso em 12 jun. 2019. 
WHITLEY, R. Changes in the social and intellectual organization of the sciences: professionalization and the arithmetic ideal. In: MENDELSOHN, E.; WEINGART, P.; WHITLEY, R. (Ed.). The social production of scientific knowledge. Dordrechet: Reidel, 1977. p.143-170. (Sociology of the sciences: a yearbook, v. 1).

WITTGENSTEIN, L. Tractatus Logico-Philosophicus. São Paulo: Editora da Universidade de São Paulo, 1994.

ZIMAN, J. M. A força do Conhecimento. São Paulo: Editora da Universidade de São Paulo, 1981.

ZIMAN, J. M. Conhecimento público. Belo Horizonte: Itatiaia; São Paulo: EDUSP, 1979.

ZIMAN, J. M. An introduction to Science studies: the philosophical and social of Science and technology. Cambrigde: University Press, 1984.

\section{APÊNDICE A - REFERÊNCIAS DOS ARTIGOS PUBLICADOS, CONFORME QUADRO 2}

SILVEIRA, M. A. A. da; CAREGNATO, S. E. Percurso histórico-epistemológico dos estudos de citação no Brasil. Transinformação, Campinas, v. 29, n. 1, p. 39-55, jan./abr., 2017. DOI: https://doi.org/10.1590/2318-08892017000100005. Acesso em: 17 jun. 2020.

SILVEIRA, M. A. A. da; CAREGNATO, S. E. Demarcações epistemológicas dos estudos de citação: concepção sociocultural das citações. Perspectivas em ciências da informação, Belo Horizonte, v. 23, n. 1, p. 55-70, jan./mar., 2018. DOI: http://dx.doi.org/10.1590/1981-5344/3125. Acesso em: 17 jun. 2020.

SILVEIRA, M. A. A. da; CAREGNATO, S. E. Demarcações epistemológicas dos estudos de citação: o fenômeno da citação. Informação \& Sociedade:

Estudos, v. 27, n. 3, 24 dez. 2017. Disponível em:

https://periodicos.ufpb.br/ojs2/index.php/ies/article/view/33847. Acesso em: 05 jun. 2020.

SILVEIRA, M. A. A. da; CAREGNATO, S. E. Demarcações epistemológicas dos estudos de citação: teorias das citações. Em Questão, Porto Alegre. v. 23, n. 3, p. 250-275, set./dez. 2017. DOI: http://dx.doi.org/10.19132/18085245233.250-275. Acesso em: 17 jun. 2020.

BUDD, J. M. Academic libraries and knowledge: a social epistemology framework. Journal of Academic Librarianship, v. 30, n. 5, p. 361-367, set. 2004. DOI: https://doi.org/10.1016/j.acalib.2004.06.002. Acesso em: 17 jun. 2020. 
BUDD, J. M; DUMAS, C. Epistemic Multiplicity in iSchools: expanding knowledge through Interdisciplinarity. Canadian Journal of Information and Library Science. v. 38, n. 4, p. 271-286, dez. 2014. DOI:

https://muse.jhu.edu/article/563461/summary. Acesso em: 17 jun. 2020.

WHITMIRE, E. Epistemological beliefs and the information-seeking behavior of undergraduates. Library \& Information Science Research. v. 25, n. 2, p. 127142, 2003. DOI: https://doi.org/10.1016/S0740-8188(03)00003-3. Acesso em: 17 jun. 2020.

WHITMIRE, E. The relationship between undergraduates' epistemological beliefs, reflective judgment, and their information-seeking behavior.

Information Processing \& Management. v. 40, n. 1, p. 97-111, jan. 2004. DOI: https://doi.org/10.1016/S0306-4573(02)00099-7. Acesso em: 17 jun. 2020.

BEZERRA, M. A. A; ARAÚJO, E. A. de. Reflexões Epistemológicas no contexto do Orkut: ética da informação, sociabilidade, liberdade e identidade.

Perspectivas em Ciência da Informação, v. 16, n. 2, p. 50-66, mar. 2011. Disponível em:

http://portaldeperiodicos.eci.ufmg.br/index.php/pci/article/view/709/924. Acesso em: 05 jun. 2020.

BRASILEIRO, F. S.; LOUREIRO, J. M. M.; FREIRE, G. H. D. Uma reflexão histórico-epistemológica da perspectiva social no campo da Ciência da Informação. Investigación Bibliotecológica: archivonomía, bibliotecología e información, v. 29, n. 65, p. 137-159, 2015. DOI:

http://dx.doi.org/10.1016/j.ibbai.2016.02.018. Acesso em: 17 jun. 2020.

CARTER, S. M.; LITTLE, M. Justifying knowledge, justifying method, taking action: epistemologies, methodologies, and methods in qualitative research.

Qualitative Health Research, v. 17, n. 10, p.1316-1328, 2007. Disponível em: https://www.researchgate.net/publication/5847019_Justifying_Knowledge_Justif ying_Method_Taking_Action_Epistemologies_Methodologies_and_Methods_in _Qualitative_Research. Acesso em: 17 jun. 2020.

$\mathrm{CHOO}, \mathrm{C} . \mathrm{W}$. Information seeking in organizations: epistemic contexts and contests, Information Research, v. 12, n. 2, 2007. Disponível em: http://informationr.net/ir/12-2/paper298.html. Acesso em 05 jun. 2020.

CORADINI, O. L. The divergences between Bourdieu's and Coleman's notions of social capital and their epistemological limits. Social Science Information, v. 49, n. 4, p. 563-583, 2010. DOI: https://doi.org/10.1177/0539018410377130. Acesso em: 17 jun. 2020.

DEMAREST, B.; SUGIMOTO, C. R. Argue, observe, assess: measuring disciplinary identities and differences through socio-epistemic discourse. Journal of the Association for Information Science and Technology, n. 66, p.1374-1387, 2015. DOl: https://dl.acm.org/doi/10.1002/asi.23271. Acesso em: 17 jun. 2020. 
FALLIS, D. Toward an epistemology of Wikipedia. Journal of the American Society for Information Science and Technology, n. 59, p.1662-1674, 2008. DOI: https://doi.org/10.1002/asi.20870. Acesso em: 17 jun. 2020.

FRANCELIN, M. M. Epistemologia da Ciência da Informação: evolução da pesquisa e suas bases referenciais. Perspectivas em Ciência da Informação, Belo Horizonte, v. 23, n. 3, p. 89-103, jul./set., 2018. DOI: https://doi.org/10.1590/1981-5344/3135. Acesso em: 17 jun. 2020.

FRICKE, M. Big Data and Its Epistemology. Journal of the Association for Information Science and Technology, v. 66, n. 4, p. 651-661, 2015. DOI: https://doi.org/10.1002/asi.23212. Acesso em: 17 jun. 2020.

FURLAN, P.K.; LAURINDO, F.J.B. Agrupamento epistemológico de artigos publicados sobre análise de big data. Transinformação, v. 29, n. 1, p. 91-100, 2017. DOl: http://dx.doi.org/10.1590/2318-08892017000100009. Acesso em: 17 jun. 2020.

FURNER, J. "A brilliant mind": Margaret Egan and social epistemology. Library Trends, v. 52, n. 4, p. 792 - 809, 2004. Disponível em: https://www.researchgate.net/publication/32956275_A_Brilliant_Mind_Margaret Egan_and_Social_Epistemology. Acesso em: 17 jun. 2020.

GOERTZ, G.; MAHONEY, J. Concepts and measurement: ontology and epistemology. Social Science Information, v. 51, n. 2, 205-216, 2012. DOI: https://doi.org/10.1177/0539018412437108. Acesso em: 17 jun. 2020.

HARTEL, J. Social Epistemology as Theoretical Foundation for Information Science: supporting a cultural Turn, Copenhagen, August 16-17, 2017. Knowledge Organization, v. 45, n. 1, p. 79-84, 2018. DOI: https://doi.org/10.5771/0943-7444-2018-1-79. Acesso em: 17 jun. 2020.

LEE, H. L. Epistemic foundation of bibliographic classification in early China: a $R u$ classicist perspective, Journal of Documentation, v. 68 , n. 3, p. 378-401, 2012. DOI: https://doi.org/10.1108/00220411211225593. Acesso em: 17 jun. 2020.

MICHELL, J. Epistemology of Measurement: the relevance of its history for quantification in the Social Sciences. Social Science Information, v. 42, n. 4, p. 515-534, 2003. DOl: https://doi.org/10.1177/0539018403424004. Acesso em: 17 jun. 2020.

MOSTAFA, S. P. Epistemologia ou filosofia da Ciência da Informação? Informação \& Sociedade: estudos, v. 20, n. 3, p. 65-73, set./dez. 2010. Disponível em: https://periodicos.ufpb.br/ojs2/index.php/ies/article/view/9048. Acesso em: 17 jun. 2020.

SEMELER, A. R.; PINTO, A. L.; VIANNA, W. B. E-Science: an epistemological analysis based on the philosophy of technology. IFLA Journal, v. 43, n. 2, p. 
198-209, jun. 2016. DOI: https://doi.org/10.1177/0340035216678235. Acesso em: 17 jun. 2020.

SVENONIUS, E. The epistemological foundations of knowledge representations, Library Trends, v. 52, n. 3, p. 571-587, 2004. Disponível em: https://www.ideals.illinois.edu/bitstream/handle/2142/1691/Svenonius571587.pd f?sequence=2\&isAllowed=y. Acesso em: 17 jun. 2020.

ZHANG, G.; JACOB, E. K. Understanding boundaries: physical, epistemological and virtual dimensions. Information Research, v. 18, n. 3, 2013. Disponível em: http://InformationR.net/ir/18-3/colis/paperC21.html. Acesso em: 05 jun. 2020.

\title{
EPISTEMOLOGY IN INFORMATION SCIENCE: INTERNATIONAL SCENARIO FROM WEB OF SCIENCE
}

\begin{abstract}
Introduction: The publication of scientific articles is necessary for the advancement of science. Objective: The general objective is to analyze the international scenario of scientific publication on Epistemology in journals in the Information Science area indexed by Web of Science (WoS). The specific objectives are a) to identify the titles of journals with the largest number of publications on the topic, as well as their editorial typification; b) report the types of access of the journals by country; c) point out the most used keywords and d) identify the epistemologists most used in the theoretical framework. Methodology: The study was characterized as exploratory, descriptive, and quantitative. As technical procedures, a bibliographic search was made in the main collection of WoS, using the search strategy: $\left(\mathrm{Tl}=\left(\right.\right.$ epistem $\left.{ }^{*}\right)$ AND $\mathrm{SU}=($ library and Information Science)). The stipulated period was from 2003 to 2018 and a total of 113 valid articles were obtained. Results: The results show that the journals with the most articles published were Knowledge Organization, with 14 articles, followed by Journal of Documentation, with nine articles. It is observed that the 113 articles were published in 41 journal titles and belong to 26 different publishers. The most used keywords were "epistemology" mentioned 23 times, followed by "information science", 10 times, and "methodology", 04 times. As for the most cited epistemologists, the highlight was Wittgenstein, followed by Kuhn, Feyerabend, and Popper. Conclusions: It is concluded that the international scenario of scientific publication on Epistemology in journals in the Information Science area, indexed by Web of Science (WoS) reports the philosophical and theoretical evolution of epistemology itself, where, still, the great majority $(73,2 \%)$ are subscription securities.
\end{abstract}

Descriptors: Scientific textual production. Scientific journals. Epistemology.

\section{EPISTEMOLOGÍA DE LA CIENCIA DE LA INFORMACIÓN: ESCENARIO INTERNACIONAL DE LA WEB OF SCIENCE}




\section{RESUMEN}

Introducción: La publicación de artículos científicos es necesaria para el avance de la ciencia. Objetivo: El objetivo general es analizar el escenario internacional de publicación científica sobre Epistemología en revistas del área de Ciencias de la Información indexadas por la Web of Science (WoS). Los objetivos específicos son a) identificar los títulos de las revistas con el mayor número de publicaciones sobre el tema, así como su tipificación editorial; b) informar los tipos de acceso de las revistas por país; c) señalar las palabras clave más utilizadas y d) identificar los epistemólogos más utilizados en el marco teórico. Metodología: El estudio se caracterizó como exploratorio, descriptivo y cuantitativo. Como procedimientos técnicos, se realizó una búsqueda bibliográfica en la colección principal de WoS, utilizando la estrategia de búsqueda: $\left(\mathrm{Tl}=\left(\right.\right.$ epistem $\left.{ }^{\star}\right) \mathrm{Y} \mathrm{SU}=($ biblioteca y ciencias de la información $\left.)\right)$. El período estipulado fue de 2003 a 2018 y se obtuvieron un total de 113 artículos válidos. Resultados: Los resultados muestran que las revistas con más artículos publicados fueron Knowledge Organization, con 14 artículos, seguidos por Journal of Documentation, con nueve artículos. Se observa que los 113 artículos fueron publicados en 41 títulos de revistas y pertenecen a 26 editoriales diferentes. Las palabras clave más utilizadas fueron "epistemology" mencionadas 23 veces, seguidas de "information science", 10 veces y "methodology", 04 veces. En cuanto a los epistemólogos más citados, lo más destacado fue Wittgenstein, seguido de Kuhn, Feyerabend y Popper. Conclusiones: Se concluye que el escenario internacional de publicación científica sobre Epistemología en revistas en el área de Ciencias de la Información, indexado por la Web of Science (WoS) informa la evolución filosófica y teórica de la epistemología en sí, donde, aún, la gran mayoría $(73,2 \%)$ son valores de suscripción.

Descriptores: Producción textual científica. Revistas científicas. Epistemología.

Recebido em: 15.01 .2020

Aceito em: 30.05 .2020 